\title{
An Empirical Study of Different Types of Changes in the Eclipse Project
}

\author{
Pitamber Tiwari, Wei Li, Raouf Alomainy ${ }^{*}$ and Bingyang Wei
}

Computer Science Department, University of Alabama in Huntsville, Huntsville, Alabama 35899, USA

\begin{abstract}
This paper studied the distribution of different types of changes in the various contexts of the system and the relationship between artifact (file and module) size and different changes. We used the change data in the open source Eclipse Project through its decade-long evolution history. The latest release has 220 modules, 33904 files, 3780201 lines of code, and 49853 changes (accumulatively). This study focused on two levels of software artifacts: module and file; and four contexts of changes: all changes, error changes, non-error changes, and 19 change categories.

At the module level, we found that the power-law distribution was a common phenomenon for three contexts of changes at both the module and file levels: it existed in all changes, in error changes, and in non-error changes. When we analyzed the 19 change categories, the files and modules exhibited different behavior: the power-law distribution existed in all but one category at the module level, but, about two third of the change categories did not show the power-law distribution at the file level.

On the relationship between artifact size and changes, we found, at the module level, a few modules that had the majority of changes accounted for the majority of the code size; however, this phenomenon disappeared when we separated the error from non-error changes. At the file level, this phenomenon did not exist at all. We did not find any correlation between artifact size and changes at either the module or file level.
\end{abstract}

Keywords: Software change distribution, large object-oriented system, Post-release evolution.

\section{INTRODUCTION}

Making changes to a large object-oriented software system is a common, difficult, and error-prone process that many software engineers face daily. Software changes are triggered by two types of events: to fix errors (bugs) and to make changes for non-error reasons, such as refactoring [1]. Understanding change characteristics in software systems, especially where they concentrate at various levels of artifacts, can help us find effective methods to handle changes.

\subsection{Software Errors}

Software error (bug) distribution has been investigated by many studies. Basili and Perricone found that the majority of errors were due to incorrect functionality specifications or requirements. They also found that $62 \%$ of the changes were due to error correction and $38 \%$ were due to modifications [2]. Shen and colleagues discovered the inverse relationship between size and number of errors up to the size of 500 lines [3]. Withrow found that the error density rose with respect to size beyond 255 lines and suggested that the testing should focus on modules of size either less than 200 lines or greater than 500 lines [4]. Moller and Paulish discovered that the changes in the source code increased error rate and the percentage of changed code and number of errors had inverse relationship [5]. Ohlsson and Alberg showed that $60 \%$ of the

*Address correspondence to these authors at the Computer Science Department, University of Alabama in Huntsville, Huntsville, Alabama 35899, USA; Tel: +1 (256) 509-1468; Fax: +1 (256) 8240-6239;

E-mail: ralomain@cs.uah.edu errors were located in $20 \%$ of the modules [6]. A study conducted by Hatton showed that the errors were concentrated in modules with 200 to 400 lines of code (LOC) [7]. Fenton and Ohlsson showed that errors followed "Pareto principle of distribution" but did not find LOC as a good predictor [8]. The study conducted by Ostrand and colleagues showed support for Pareto principle in errors and discovered LOC as the strongest predictor [9]. Andersson and Runeson showed that errors followed "Pareto principle of fault distribution" and size metrics were good predictor of pre-release errors in modules [10]. Zhang discovered that Weibull probability distribution function described the error distribution more precisely [11]. El Emam and colleagues studied a large C++ system and found the confounding effect of class size on the prediction of software faults, and suggested that class size was a major predictor of class faults [12].

These studies suggested that Pareto principle was a common phenomenon for software errors, whereas the relationship of artifact (class, file, or module) size and errors was less clear. Our research objective is to verify the results from the previous error distribution studies by using the data from a large open source system: Eclipse, in its decade long evolution history.

\subsection{Error Categories}

Although there have been many studies on software errors, few have analyzed software errors in detailed categories. The Institute of Electrical and Electronics Engineers (IEEE) published the Standard Classification for Software Anomalies [13] that introduced error categories. Other error 
classifications were introduced by Li and colleague [14], Lo and colleagues [15], and Pan and colleagues [16] .

Previous work has focused on error vs. no error. Our research objective is to classify errors into more detailed categories and verify the research results from previous studies. Knowing where each type of error concentrates will help us develop more focused methods that avoid introducing them or find a certain type of errors in software. For example, if we know that the memory-related errors: memory leaks, dangling pointers, and array-out-of-bounds, are concentrated in a small set of modules that share certain characteristics, we can develop tools that monitor the module characteristics and alert programmers to watch out for memory-related errors when these modules are being developed or maintained, thus reducing memory errors in code. This knowledge also allows us to be more precise about where and how to find certain types of errors. For example, we can increase the testing effort to hunt for memory errors when those modules are identified and tested.

\subsection{Non-Error Changes}

Often, software engineers make changes to software systems for non-error reasons (such as refactoring). The studies on changes that were not caused by fixing errors were scarce. Understanding where non-error changes concentrate is not less important to that of errors, because changes, regardless of the triggers, are prone to errors and affect software quality. Soares and colleagues in their study [17] presented a technique to identify issues, mainly on semantics, which resulted from non-error refactoring effort. Görg and Weißgerber showed how incomplete refactoring can cause long standing bugs [18].

\subsection{Is Software Size an Effective Predictor of Error Rate}

Many previous studies investigated whether the size of a module is an effective predictor of the module's error rate [5, $9,10,12,19,20]$. The majority of these studies found the two correlated. Our research goal in this aspect is to investigate if a correlation between the two exists in the large open source system's evolution history.

\subsection{The Scope of this Research Study}

In this study, we have investigated the power-law distribution (Pareto principle) in many contexts: at module level, a file level, for all changes, for error changes, for non-error changes, and for each of the 19 change categories. We also examined the relationship between changes and artifact size in the same contexts. We studied 220 modules, 33,904 files, and a total of 3,780,201 lines of code (LOC) - the total number of lines in the source code excluding the comments and blank spaces - and 5,908,044 physical lines - the total lines in the source code including the blank lines, single braces or parentheses, - and a total of 49,853 unique change identifiers in the Eclipse Project's evolution history that spanned more than a decade.

\subsection{Research Objectives}

The objective of this research is twofold: to study the change distribution and to investigate the relationship between changes and artifact size. We are interested in verifying the Pareto principle in change distribution, that is, a small percentage of artifacts (modules and files) contain the majority of changes (all changes, error changes, non-error changes, and each category of changes). The following are the research hypotheses:

Hypothesis \#1: Changes have the power-law distribution at the module and file level.

Hypothesis \#2: Both error changes and non-error changes follow the power-law distribution.

Hypothesis \#3: Changes in each classification category follow the power-law distribution.

Hypothesis \#4: The artifact size is correlated with changes in the artifact.

Hypothesis \#5: Modules and files with majority of changes contain most of the code size.

\section{MATERIALS AND METHODOLOGY}

Eclipse is a large composite open source software system that is made of many subsystems, primarily written in the Java programming language. Each subsystem is made of several projects. Out of several Eclipse subsystems, we focused on the Eclipse Project subsystem. Eclipse Project consists of five projects: e4, Eclipse Project Incubator (EPI), Java Development Tool (JDT), Plugin Development Environment (PDE), and Eclipse Platform (EP). JDT, PDE, and EP are further divided into subprojects. Each subproject comprises of modules and module alias. A module is made of source files. A module alias is a virtual module that is made of source files that come from other modules. For example, Module A has two source files: A1.java and A2.java, and Module B has two source files: B1.java and B2.java. A module alias MA may be made of A1.java and B2.java. Every project or sub-project under Eclipse Project is a combination of several modules and/or module aliases. We only analyzed modules in this study because module alias did not contribute any new files to the system.

\subsection{The CVS and Bugzilla Repositories}

Eclipse uses Concurrent Versioning System (CVS) repository to keep track of changes in project files [21]. CVS is a version control system that maintains history of the files throughout their development and evolution.

We created a crawler tool to extract information from the Java files located in the Eclipse CVS repository. To classify each error change to a specific error category, we tried the change description field from the CVS repository first. The change descriptions (known as bug descriptions in CVS and Bugzilla) extracted from the CVS repository were the titles of the changes. They did not have enough information to describe a change completely for classification purpose. On several runs, we found the change descriptions to be misleading and inappropriate, sometimes even empty. To mitigate the problem of insufficient information in the CVS repository, we went to Bugzilla, another change repository for the Eclipse system, which had more detailed change description for each change identifier (bugID) that we found in the CVS repository. The crawler tool extracted the module name, file name, revision, bug number (bugID), and bug description from the CVS repository and then used the bugID to extract the detailed description of the changes from the Bugzilla. 


\subsection{Change Categories}

We classified the changes into 19 specific categories and studied the change distribution in each category. The change categories that we adopted came from the combination and customization of the categories suggested and used by Li and colleagues [14], Pan and colleagues [17], Lo and colleagues [15], and IEEE Standard Classification for Software Anomalies [13]. Table 1 summarizes the change categories that we used in our study.

\section{Table 1. List of Change Categories}

\begin{tabular}{|c|c|}
\hline Bug Category & Description \\
\hline Adaptive Maintenance & $\begin{array}{l}\text { A type of maintenance performed } \\
\text { to change software so that it will work in an } \\
\text { altered environment, such as when } \\
\text { an operating system, hardware platform, } \\
\text { compiler, software library or database } \\
\text { structure changes, compatibility changes, etc. }\end{array}$ \\
\hline Enhancement & $\begin{array}{l}\text { Actions performed to add a new capability or } \\
\text { improve the existing capability to software; } \\
\text { Bugs that cause failure to meet the perform- } \\
\text { ance requirements of the product. } \\
\text { Examples: } \\
\text { Functions correctly but runs/respond slowly. } \\
\text { Takes longer time to perform a task. } \\
\text { Addition of a new feature. }\end{array}$ \\
\hline Not Bugs & $\begin{array}{l}\text { Test cases, simple updates. } \\
\text { Example: } \\
\text { File name update, copy right update, and test } \\
\text { case addition. }\end{array}$ \\
\hline Refactoring & $\begin{array}{l}\text { Changes of source code without modifying } \\
\text { the functional behavior to improve some } \\
\text { nonfunctional attributes, such as readability, } \\
\text { reduced complexity, or maintainability, of the } \\
\text { software. }\end{array}$ \\
\hline Coding & $\begin{array}{l}\text { Bugs in the decision logic, branching, se- } \\
\text { quencing, computational logic, and typos in } \\
\text { programming. } \\
\text { Examples: } \\
\text { Wrong concept employed in coding. } \\
\text { Navigation not coded correctly in source code. } \\
\text { The control flow is incorrectly implemented. } \\
\text { Incorrect processing and evaluation of expres- } \\
\text { sion and equations. }\end{array}$ \\
\hline Concurrency & $\begin{array}{l}\text { Bugs that happen in multithreading or multi- } \\
\text { process environment, including data race, } \\
\text { deadlock and synchronization. }\end{array}$ \\
\hline Data & $\begin{array}{l}\text { Bugs in definition, structure, mapping, access, } \\
\text { scope, use, or initialization. } \\
\text { Examples: } \\
\text { Incorrect object type or dimension. } \\
\text { Incorrect initial default values. } \\
\text { Incorrect duplication or failure to create a } \\
\text { duplicate object. } \\
\text { Incorrect access to object. } \\
\text { Use of incorrect variable names. }\end{array}$ \\
\hline Documentation & $\begin{array}{l}\text { Bugs in the specifications and Java docu- } \\
\text { ments. } \\
\text { Bugs in program help. }\end{array}$ \\
\hline
\end{tabular}

\begin{tabular}{|c|c|}
\hline Functionality & $\begin{array}{l}\text { Function is incorrect, ambiguous, inconsis- } \\
\text { tent, missing, incomplete, that do not need to } \\
\text { be present, or do not behave as expected. } \\
\text { Bugs related to core functionality implemen- } \\
\text { tation. Functions that do not meet the specifi- } \\
\text { cation requirement or the standards with the } \\
\text { software version, or coding conventions, } \\
\text { representation. Any other semantic bug that } \\
\text { does not meet the design requirements. } \\
\text { Examples: } \\
\text { Unnecessary features. } \\
\text { Duplicate features. } \\
\text { Misplaced features. } \\
\text { Missing Validation. }\end{array}$ \\
\hline Generic & $\begin{array}{l}\text { Unknown bugs. } \\
\text { Bugs related to security. } \\
\text { Bugs related to scalability. } \\
\text { Bugs with missing bug description. }\end{array}$ \\
\hline GUI & $\begin{array}{l}\text { Bugs related to graphical user interface and } \\
\text { CSS bugs. } \\
\text { Examples: } \\
\text { Incorrect alignment of components. } \\
\text { Incorrect size and shape of interface. } \\
\text { Incorrect layout of reports. } \\
\text { Incorrect coloring. }\end{array}$ \\
\hline $\begin{array}{l}\text { Handling } \\
\text { Event/Exception }\end{array}$ & $\begin{array}{l}\text { Missing event handling or improper event } \\
\text { handling. } \\
\text { Do not have proper exception handling. } \\
\text { Anomalies caused by exception. } \\
\text { Examples: } \\
\text { Null pointer exception. } \\
\text { Class cast exception. } \\
\text { Missing key press or mouse movement han- } \\
\text { dling. } \\
\text { Incorrect mouse click, hover, and double click } \\
\text { handling. } \\
\text { Incorrect action selection. } \\
\text { Timer expiration. }\end{array}$ \\
\hline $\mathrm{I} / \mathrm{O}$, Serialization & $\begin{array}{l}\text { Bugs related to I/O handling, import and } \\
\text { export of files, serialization of objects. } \\
\text { I/O-related to read from and write to files, } \\
\text { memories, sockets. }\end{array}$ \\
\hline $\begin{array}{l}\text { Internationalization: } \\
\text { Localization with } \\
\text { Resource Bundles }\end{array}$ & $\begin{array}{l}\text { Bugs due to adaption to various languages and } \\
\text { regions without engineering changes. Bugs } \\
\text { due to adaption of internationalized software } \\
\text { for a specific region or language. }\end{array}$ \\
\hline Memory & $\begin{array}{l}\text { Bugs related to memory. } \\
\text { Examples: } \\
\text { Buffer overflow. } \\
\text { Memory access violations. } \\
\text { Memory leak. } \\
\text { Dangling pointer. } \\
\text { Null pointer dereference } \\
\text { Double free. }\end{array}$ \\
\hline Message & $\begin{array}{l}\text { Defects in message and logs, inadequate, } \\
\text { incorrect, misleading, or missing bug mes- } \\
\text { sages in source code or bug logs. }\end{array}$ \\
\hline Regression/rollback & $\begin{array}{l}\text { Bugs due to changes in some function or } \\
\text { code, change rollback, and changes not made }\end{array}$ \\
\hline
\end{tabular}




\begin{tabular}{|l|l|}
\hline & $\begin{array}{l}\text { properly. } \\
\text { Examples: } \\
\text { Incorrect merging of codes. } \\
\text { You commit a fix, but it did not work so the } \\
\text { changes have to be rolled back. }\end{array}$ \\
\hline Third Party & Bugs due to third party software. \\
\hline
\end{tabular}

The top four categories are non-error changes, whereas the remaining categories are all error categories.

\subsection{Classification and Conflict Resolution}

Change categorization or classification is a difficult task. Sometimes, a change could be classified into several different categories. There seems to be no universally correct way to categorize changes to a particular change category. Change categories, literally, can be infinite [22].

We tried the automated text (change) classification techniques of the Support Vector Machine [20] and the Naïve Bayes [19] and the tool provided by Dr. Paul Wofgang [23], and found that the classification accuracy was not acceptable. Therefore, we performed the change classification manually. Based on the history information, change description, comments, reviews from developers, each change (bugID) was placed in only one category. We tried to resolve the conflicts based on the conscience of the comments in Bugzilla. When a bug could not be placed in a known category, it was placed in the Generic category. When a nonerror change could not be placed in any of the three clearly defined non-error categories, it was placed in the Not Bugs category.

Out of the 220 modules that we examined, 52 modules were change free. We classified the changes in 140 modules, which accounted for $83.3 \%$ of the modules that had changes.

\section{ARTIFACT SIZE AND CHANGES}

To analyze the relationship between artifact size and changes, we used Resource Standard Metrics (RSM), a source code metrics and quality analysis tool, to get the size metrics. For our study, we used the tool's default source code size metrics for modules and files. The source code size metrics available from the tool are the Lines of Code (LOC), Effective Lines of Code (eLOC), Logical Lines of Code (lLOC), Comment, and Lines.

$L O C$ is the total number of lines in the source code excluding the comments and blank lines. $e L O C$ gives the count of the total number of source code lines that are not comments, blank lines, standalone parenthesis or braces. The eLOC metric is a result of subtracting total number of single braces or parenthesis from the LOC metric. Many programmers put single brace or parenthesis on a new line to make source code clean and readable. This programming style leads to the increase of the LOC metric, but not eLOC.

$I L O C$ represents the total number of lines in the source code that are terminated by a semicolon. The 'for' loop control statement contains two semicolon, however it accounts for only one in the calculation of the ILOC metric.

Comment represents the total lines of comments in the source code. This metric is a general measure of the effort by the developer to make the program more understandable.

Lines is the total lines in the source code including the blank lines, single braces, or parenthesis. It is sometimes called Physical Lines of Code.

\subsection{Metric Calculation}

We use an example, adapted from the documentation of RSM, to show how the RSM tool calculated the metrics. In Table 2 , the presence of check mark $(\checkmark)$ indicates the particular line in the sample source code is included in the count of the respective metrics. The table indicates that the tool considered four lines of source code for the calculation of $L O C$, two lines of source code for the calculation of $e L O C$, one line of source code for the calculation of $1 \mathrm{LOC}$, two lines of source code for the calculation of Comment, and six lines of source code for the calculation of Lines.

\section{RESEARCH DESIGN}

To test the hypotheses, we collected the change data from 220 modules that contained 33,904 files from the Eclipse Project. The number of changes ranged from 0 to 6365 per module and from 0 to 202 per file. The total number of changes in modules and files was 49,853. Modules and files had sizes ranging from 20 LOC to 405,951 LOC per module and 1 to 48,596 LOC per file.

The power-law distribution [24] occurs in many situations of software development and evolution. The study conducted by Potanin and colleagues [25] illustrated the powerlaw distribution in the incoming and outgoing references in object graphs. Wheeldon and Counsell [26] confirmed the power-law distribution in object-oriented class relationships. Hatton [27] illustrated the power-law distribution in the equi-

Table 2. An Example Showing the Process Used by RSM to Determine the Size Metrics

\begin{tabular}{|c|c|c|c|c|c|}
\hline Source Code & LOC & eLOC & ILOC & Comments & Lines \\
\hline\{ & $\checkmark$ & & & & $\checkmark$ \\
\hline \multirow[t]{2}{*}{ //assign the grade } & & & & $\checkmark$ & $\checkmark$ \\
\hline & & & & & $\checkmark$ \\
\hline$\}$ & $\checkmark$ & & & & $\checkmark$ \\
\hline
\end{tabular}


Table 3. Distribution of Change Percentage Over Module Percentage

\begin{tabular}{|c|c|c|c|}
\hline \# of Modules & $\%$ of Modules & \# of Changes & $\%$ of Changes \\
\hline 22 & 10 & 40275 & 80.79 \\
\hline 33 & 15 & 43997 & 88.25 \\
\hline 44 & 20 & 45838 & 91.95 \\
\hline 66 & 30 & 47949 & 96.18 \\
\hline 77 & 35 & 48572 & 97.43 \\
\hline 88 & 40 & 48975 & 98.24 \\
\hline 99 & 45 & 49268 & 98.83 \\
\hline 143 & 65 & 49806 & 99.91 \\
\hline 154 & 70 & 49835 & 99.96 \\
\hline 165 & 75 & 49850 & 99.99 \\
\hline 168 & 76 & 49853 & 100.00 \\
\hline
\end{tabular}

librium component size of a system. These studies suggested that the power-law distribution was a common phenomenon in software systems.

Relatively small numbers of modules or files are believed to have most of the bugs in software systems [5, 7, 8, 10]. This idea can be summed up by the Pareto Principle [28], also known as the 20-80 rule. The power-law and Weibull distribution are commonly used distributions to confirm the Pareto Principle.

We used the Alberg Diagram, as suggested by Fenton and Ohlsson [8], and used by Timea and Barbara [29] and Anderson and Runeson [10], for testing Hypothesis \#1. We sorted the modules and files in decreasing order with respect to the number of changes. We plotted the accumulative percentage of changes on the y-axis of the Alberg Diagram and the accumulative percentage of the modules or files on the $\mathrm{x}$ axis. The Alberg Diagram provides the visual identification of a possible power-law distribution. To be certain, we used the function built in Matlab [30] as described by Aaron and colleagues [31], to test the power-law distribution at the module and file level.

To test Hypothesis \#4, we calculated the Pearson correlation coefficient [24] between the size metrics and the changes at the module and file level.

To test Hypothesis \#5, we computed and analyzed the sizes of modules and files that were responsible for a large percentage ( $80 \%$ for example) of the changes. We sorted the modules and files in decreasing order relative to the number of changes, and then plotted the accumulative percentage of changes and the LOC metric on the y-axis with respect to the accumulative percentage of modules or files on the $\mathrm{x}$-axis to see if the two curves were close.

\section{DATA ANALYSIS RESULTS}

In this section, we present the results of the hypothesis testing. In testing Hypothesis \#1, we show both the visual plots and the statistical tests to illustrate how we analyzed the data. In subsequent hypothesis tests, we only show the summary of the statistical tests to save the space.

Hypothesis \#1: Changes have the power-LAW distribution at the module and file level.

We collected 49,853 bugIDs (the unique identifiers for the changes) that affected 220 modules in the Eclipse Project. The modules were sorted in descending order with respect to the number of changes. Table 3 summarizes the distribution data for the modules.

\subsection{The Alberg Diagram for Modules}

Fig. (1) shows the Alberg Diagram for the distribution data in Table $\mathbf{3}$. The accumulative percentage of changes is on the $y$-axis and the accumulative percentage of modules is on the $\mathrm{x}$-axis.

At the module level, $20 \%$ of the modules were responsible for about $92 \%$ of the changes, and $76.36 \%$ of the modules were responsible for almost $100 \%$ of the changes. Roughly a quarter of the modules did not report any changes. Fig. (1) shows the existence of the Pareto principle and the possibility of the power-law distribution.

\subsection{Power-law Distribution for Modules Using the Statis- tical Tool}

Aaron and colleagues provided several Matlab functions to test the power-law distribution [30]. We used the plfit( $x$ ) function, which estimated the lower cutoff value $x$ min and $\alpha$ 


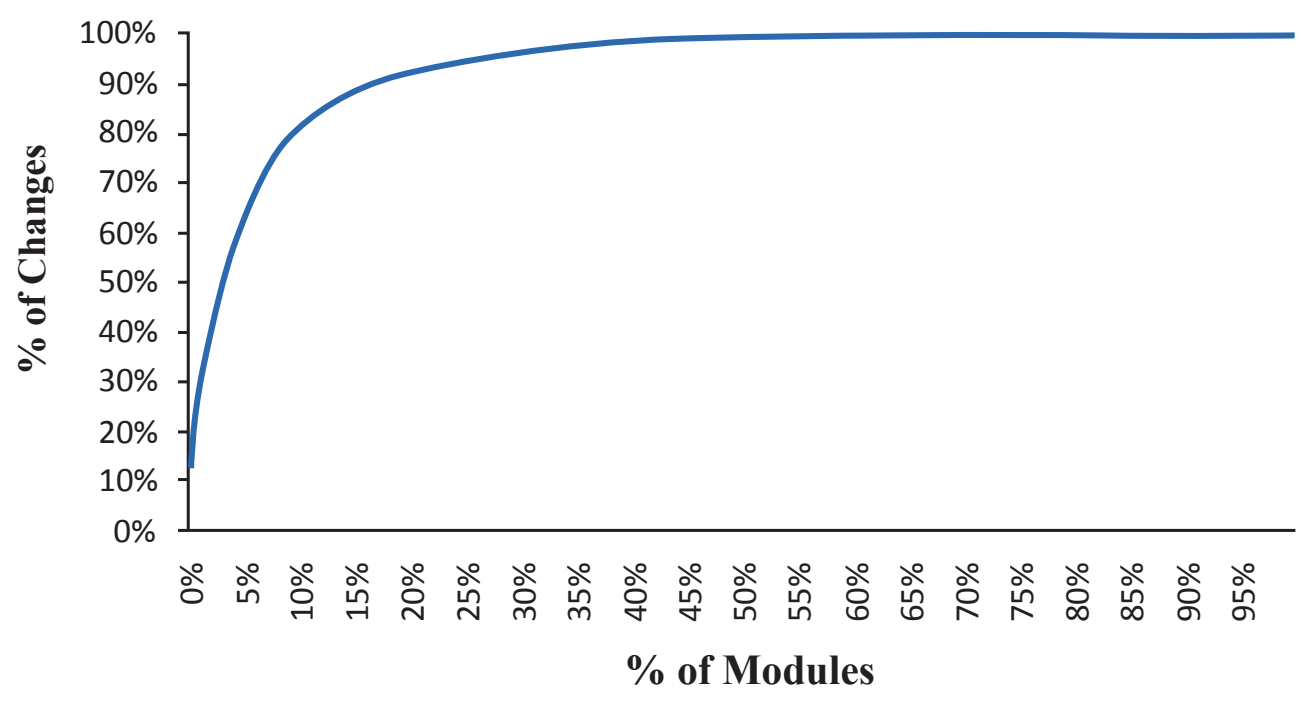

Fig. (1). Percentage of modules versus percentage of changes.

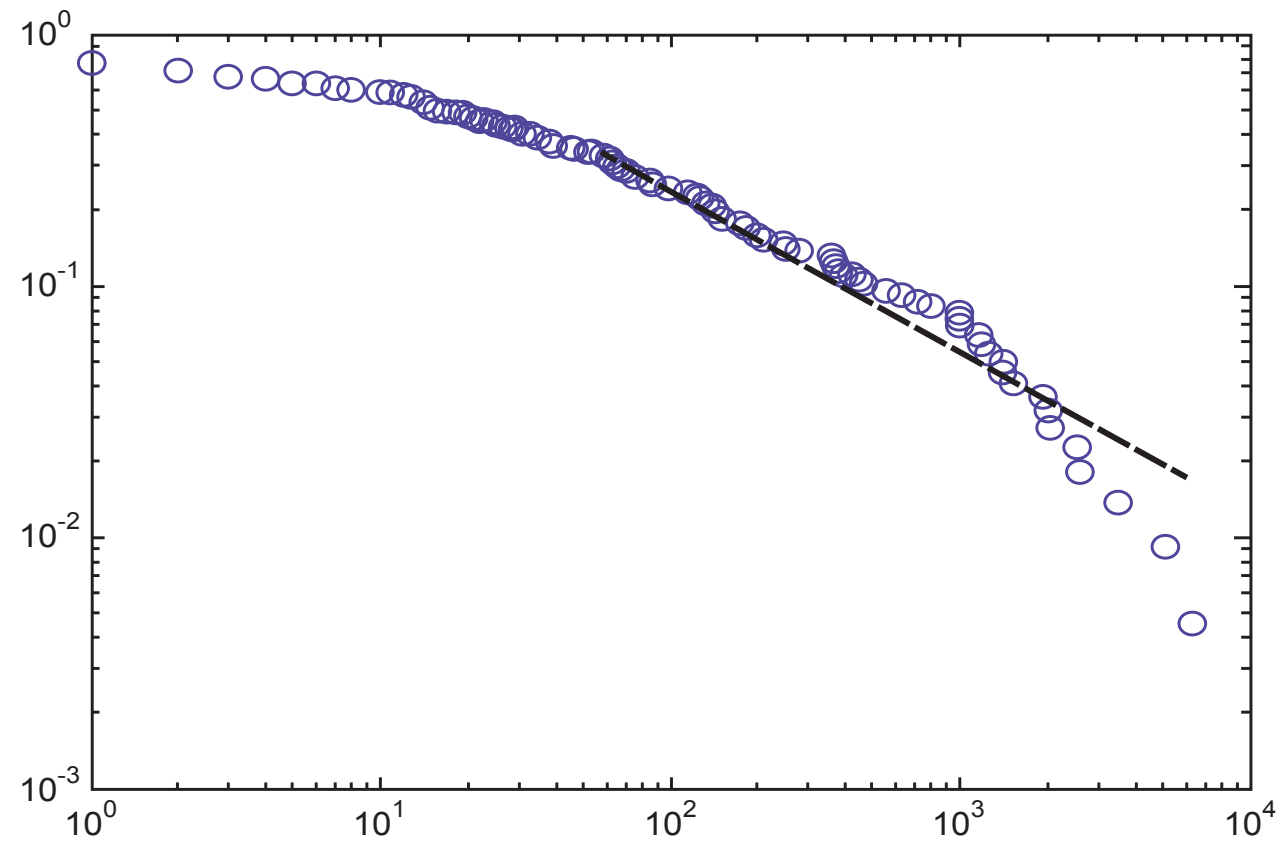

Fig. (2). Plot of the number of changes per module and power-law distribution for $\mathrm{x}$ greater than or equal to xmin.

(alpha) by implementing the maximum likelihood estimator and the goodness-of-fit. This function used the number of changes (x), given in Table $\mathbf{3}$, as input. The calculated lower cutoff value (by the function) xmin was 53. To visualize the fitted distribution, we used the plplot $(x, x m i n$, alpha) function, where $\mathrm{x}$ is the number of changes per module and $\mathrm{xmin}$ and $\alpha$ are the values computed by the plfit function. This function plotted the data and the fitted power-law distribution in log axes in Fig. (2), where the dashed line is the power-law distribution and the circled line is the module data. Fig. (2) shows that most of the module data fell on the line given by the power-law distribution.

We used the function plpva(x, xmin) that took the number of changes in modules (x) and lower cutoff value xmin to calculate the $p$-value for the Kolmogorov-Smirnov test [24]. This function estimated the p-value over many repetitions. The default number of repetitions was 1000 , which was the number that we used. The p-value computation was a slow process. In our case, it took hours to compute one p-value. The time was dependent on the number of samples and the value of each sample. The $p$-value, for 1000 repetitions, computed by the function for the number of changes in modules was 0.049 . We used the threshold value of 0.05 suggested by Aaron and colleagues [30]. If the computed p-value is greater or equal to 0.05 , the distribution follows the power law; otherwise, it does not follow. The p-value 0.049 was close enough to 0.05 , so we treated it as being equal to 0.05 and accepted the Hypothesis \#1 for the modules and concluded that the total changes (error and non-error changes together) followed the power-law distribution at the module level.

The p-value that we used thereafter is the p-value suggested by Aaron and colleagues [30] and the tools implementing their work. This p-value is different from the $\mathrm{p}$ value commonly used to reject null form of a hypothesis. 
Table 4. Distribution of Changes in Files

\begin{tabular}{|c|c|c|c|}
\hline \# of Files & \% of Files & \# of Changes & \% of Changes \\
\hline \hline 1696 & 5.00 & 30149 & 60.48 \\
\hline 3391 & 10.00 & 38443 & 77.11 \\
\hline 3871 & 11.41 & 39883 & 80.00 \\
\hline 5086 & 15.00 & 42911 & 96.08 \\
\hline 6781 & 20.00 & 45820 & 91.91 \\
\hline 10172 & 25.00 & 47515 & 95.31 \\
\hline 10814 & 30.00 & 49211 & 98.71 \\
\hline
\end{tabular}

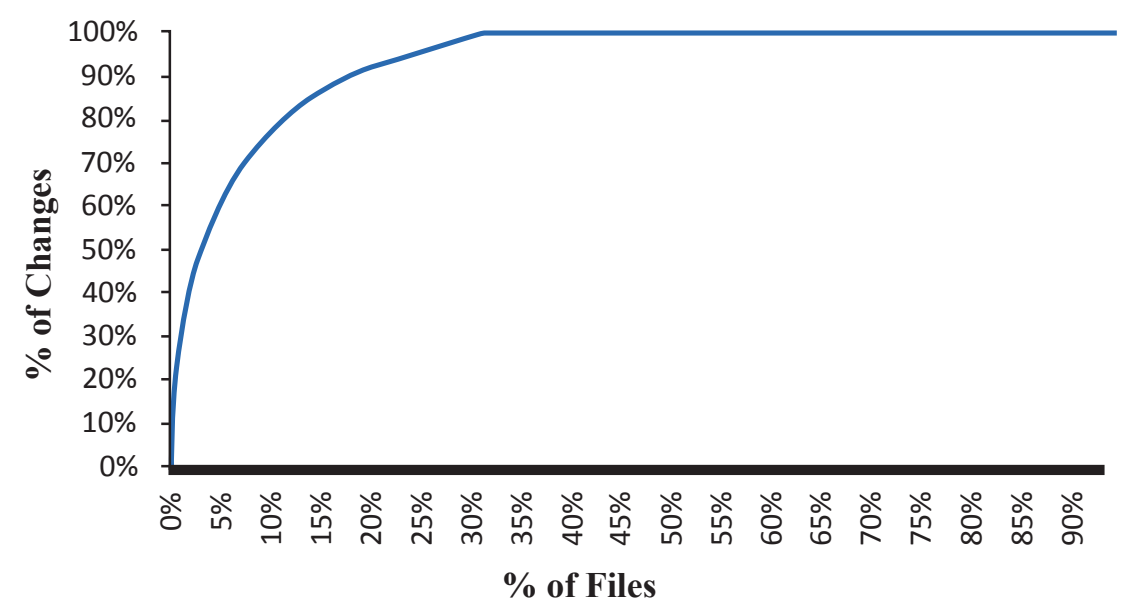

Fig. (3). Percentage of files versus percentage of changes.

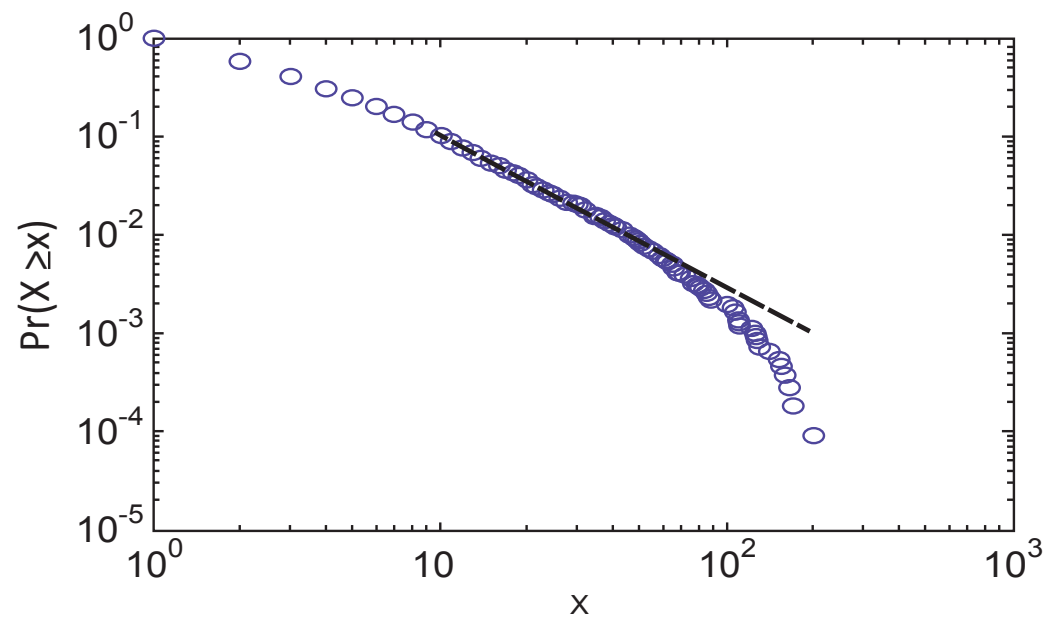

Fig. (4). Plot of number of changes per file and the power-law distribution for $\mathrm{x}$ greater than or equal to $\mathrm{xmin}$.

\subsection{The Alberg Diagram for Files}

Table 4 summarizes the data for files. We did the same tests for files as we did for modules.

Fig. (3) shows the Alberg Diagram for the distribution of changes at the file level. At the file level, $20 \%$ of the files were responsible for more than $90 \%$ of the changes. Approximately $32 \%$ of the files had $100 \%$ of the changes; that is, about two-third of the files did not report any changes.

\subsection{Power-Law Distribution for Files Using the Statisti- cal Tool}

Fig. (4) shows the power-distribution plot for the file data. Visually, we can see that the file data followed the power-law distribution line very closely. The calculated pvalue was 0.106 , which was greater than the threshold value of 0.05 . Therefore, we accepted Hypothesis \#1 for files and concluded that the total changes at the file level followed the power-law distribution. 
Table 5. The p-Values for the Error and Non-Error Changes

\begin{tabular}{|c|c|c|}
\hline p-value & Error Changes & Non-Error Changes \\
\hline \hline Modules & 0.56 & 0.99 \\
\hline Files & 0.11 & 0.45 \\
\hline
\end{tabular}

Table 6. Distribution of Changes in Each Category at the Module Level

\begin{tabular}{|c|c|c|c|c|}
\hline Category & P value & $X$ min. & \# of Modules Affected & Total Changes \\
\hline Adaptive & 0.032 & 5 & 50 & 474 \\
\hline Cleanup & 0.738 & 2 & 43 & 553 \\
\hline Coding & 0.312 & 13 & 59 & 667 \\
\hline Concurrency & 0.925 & 5 & 32 & 542 \\
\hline Data & 0.270 & 2 & 44 & 583 \\
\hline Doc & 0.585 & 8 & 32 & 239 \\
\hline Enhancement & 0.146 & 12 & 93 & 5459 \\
\hline Functionality & 0.052 & 14 & 95 & 3236 \\
\hline Generic & 0.176 & 5 & 86 & 1313 \\
\hline Gui & 0.116 & 1 & 28 & 443 \\
\hline Handling & 0.492 & 8 & 69 & 1285 \\
\hline $\mathrm{I} / \mathrm{O}$ & 0.167 & 8 & 3 & 255 \\
\hline Internationalization & $\mathrm{n} / \mathrm{a}$ & $\mathrm{n} / \mathrm{a}$ & 31 & 10 \\
\hline Memory & 0.254 & 6 & 28 & 254 \\
\hline Messaging & 0.525 & 4 & 46 & 520 \\
\hline Not bug & 0.788 & 20 & 81 & 1553 \\
\hline Refactoring & 0.882 & 7 & 66 & 1751 \\
\hline Regression & 0.409 & 2 & 40 & 272 \\
\hline Third Party & $\mathrm{n} / \mathrm{a}$ & $\mathrm{n} / \mathrm{a}$ & 6 & 10 \\
\hline
\end{tabular}

Since the changes at the module and file level followed the power-law distribution, we accepted the Hypothesis \#1.

Hypothesis \#2: Both error changes and non-error changes follow the power-law distribution.

To study the change distribution in each change category, we had to classify the changes into different categories. We tried an automated classification of the changes using data mining tools and the change descriptions that we collected from the CVS and Bugzilla repositories. Despite various attempts and different techniques, we could not get more than $65 \%$ accuracy of the automated classification. To ensure the quality of the classification and the confidence in the research results, we classified the changes manually. Out of the 49853 changes, we classified 19419 (about 40\%) of them that affected $83.3 \%$ of the modules into 19 change categories. The manual classification was very time consuming, but yielded the most accurate classification possible. The changes that were classified were randomly chosen. Although we did not classify all the changes, we believe that enough changes were classified to give us an accurate ac- count of the distribution. Of the 19 change categories, we merged all the non-error categories into one group and all the error categories into another. There were 9237 non-error changes and 10,182 error changes in modules, or $47.57 \%$ of the changes were non-error changes and $52.43 \%$ of the changes were error changes.

Table 5 summarizes the test results for the power-law distribution at the module and file level using the two groups. All p-values were greater than the threshold 0.05; therefore, we accepted Hypothesis \#2 and concluded that error and non-error changes followed the power-law distribution at the module and file level.

Hypothesis \#3: Changes follow the power-law distribution in each classification category.

Table 6 summarizes the p-values calculated for each change category at the module level; Table 7 is for files. The Matlab functions did not report the p-value for the Internationalization and Third Party categories, because there were less than 50 changes ( 50 was used as the cutoff value by the functions) in the two categories. 
Table 7. Distribution of Changes in Each Category at the File Level

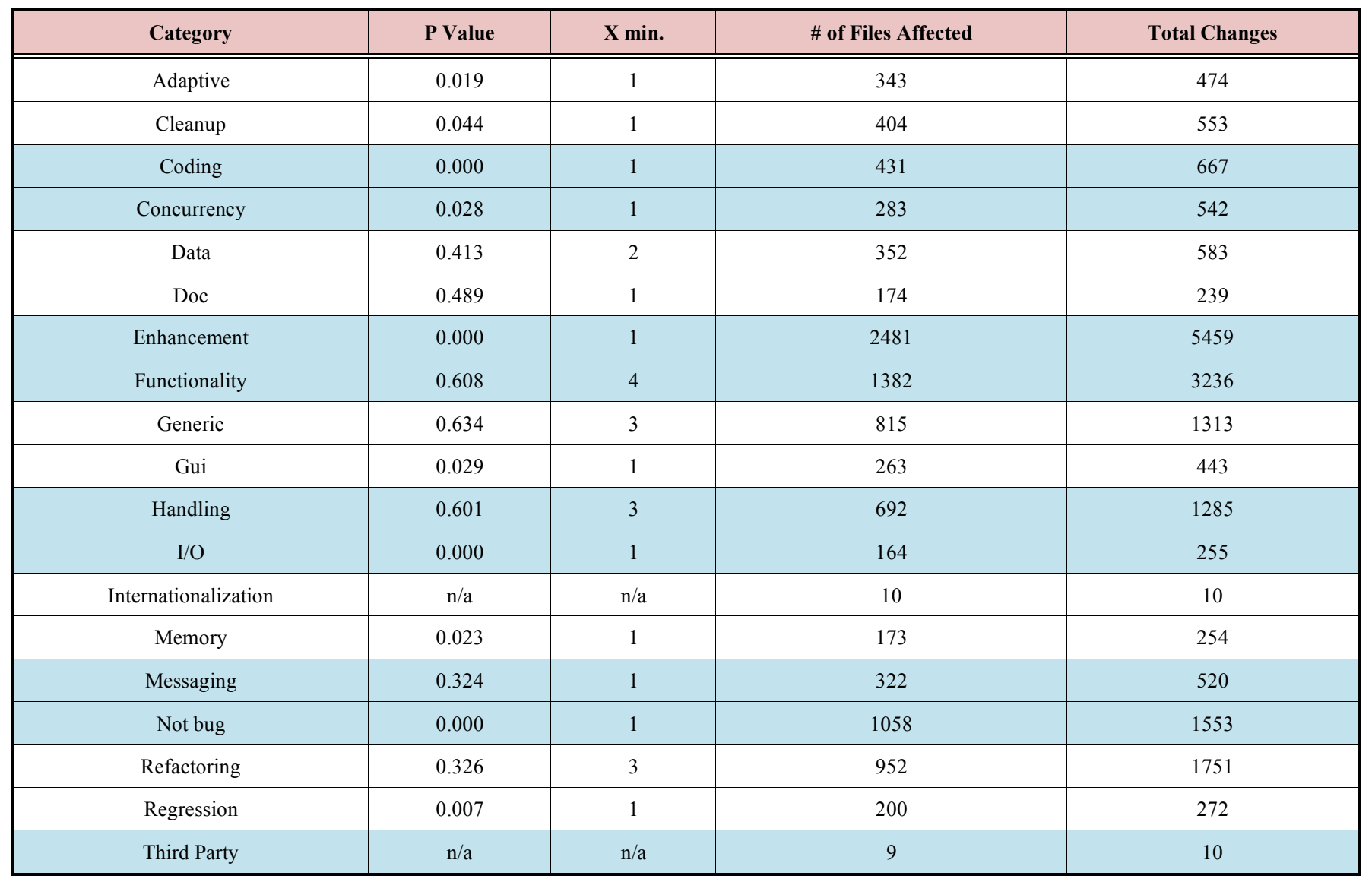

Table 8. Spearman Correlation Coefficient Values for Modules and Files

\begin{tabular}{|c|c|c|}
\hline Metrics & Modules & Files \\
\hline \hline LOC & 0.63 & 0.36 \\
\hline eLOC & 0.63 & 0.37 \\
\hline ILOC & 0.63 & 0.38 \\
\hline Comment & 0.65 & 0.42 \\
\hline Line & 0.64 & 0.42 \\
\hline
\end{tabular}

At the module level, all but one, Adaptive, categories had the $\mathrm{p}$-value greater than 0.05 . Therefore, we concluded that most but not all change categories followed the power-law distribution at the module level.

At the file level, close to two-third of the categories had the p-value less than 0.05; therefore, we concluded that most change categories did not follow the power-law distribution at the file level.

For Hypothesis \#3, we obtained the mixed results: the modules and files exhibited different behaviors when changes were examined in each category. A detailed discussion on this difference is presented in the Discussion of the Findings section.

Hypothesis \#4: The artifact size is correlated with the changes in the artifact.
Table 8 shows the Spearman correlation coefficient values between the LOC, eLOC, ILOC, Comment, and Lines metrics and the number of changes in the modules and files. These coefficients were very low and not statistically significant. Therefore, we rejected Hypothesis \#4 and concluded that there was no correlation between artifact size and the changes.

Hypothesis \#5: Modules and files containing majority of changes contain most of the code size (LOC).

\subsection{LOC for Modules}

In order to test this hypothesis, we calculated the LOC for each module using the RSM tool. There were 3,780,201 LOC across 220 modules. Table 9 summarizes the LOC data for the modules. 
Table 9. Distribution of Accumulative Percentage of Changes and LOC for the Modules

\begin{tabular}{|c|c|c|c|c|c|}
\hline \# of Modules & $\%$ of Modules & \# of Changes & $\%$ of Changes & LOC & $\%$ of LOC \\
\hline 11 & 5 & 30505 & 61.19 & 955419 & 25.27 \\
\hline 22 & 10 & 40275 & 80.79 & 1970302 & 52.12 \\
\hline 33 & 15 & 43997 & 88.25 & 2385225 & 63.10 \\
\hline 44 & 20 & 45838 & 91.95 & 2569486 & 67.97 \\
\hline 55 & 25 & 47138 & 94.55 & 3040524 & 80.43 \\
\hline 66 & 30 & 47949 & 96.18 & 3135854 & 82.95 \\
\hline 77 & 35 & 48572 & 97.43 & 3135931 & 85.00 \\
\hline 88 & 40 & 48975 & 98.24 & 3136019 & 86.77 \\
\hline 99 & 45 & 49268 & 98.83 & 3136118 & 88.14 \\
\hline 110 & 50 & 49475 & 99.24 & 3136228 & 89.56 \\
\hline 121 & 55 & 49626 & 99.54 & 3136349 & 90.50 \\
\hline 132 & 60 & 49746 & 99.79 & 3136481 & 91.48 \\
\hline 143 & 65 & 49806 & 99.91 & 3136624 & 93.44 \\
\hline 154 & 70 & 49835 & 99.96 & 3136778 & 94.58 \\
\hline 165 & 75 & 49850 & 99.99 & 3136943 & 95.32 \\
\hline 168 & 76.36 & 49853 & 100.00 & 3137111 & 95.33 \\
\hline
\end{tabular}

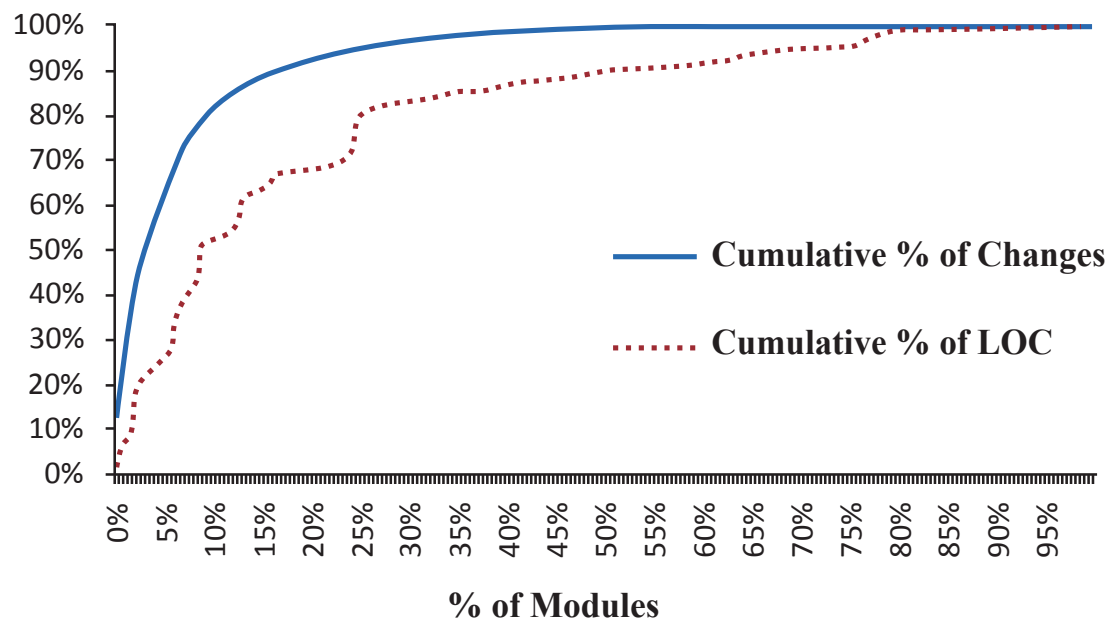

Fig. (5). Accumulative percentage of changes and LOC versus the accumulative percentage of modules.

Table 10. Distribution of Accumulative Percentage of Error Changes and LOC for the Modules

\begin{tabular}{|c|c|c|c|c|c|}
\hline \# of Modules & \% of Modules & \# of Error Changes & \% of Error Changes & LOC & 879895 \\
\hline \hline 10 & 5.20 & 6677 & 65.58 & 1067303 \\
\hline 20 & 10.40 & 8073 & 79.29 & 1465085 \\
\hline 30 & 15.60 & 8913 & 87.54 & 1633979 \\
\hline 40 & 20.80 & 9417 & 92.49 & 1701178 \\
\hline 50 & 26.00 & 9649 & 94.77 & 1740594 \\
\hline 60 & 31.20 & 9818 & 96.43 & 1788810 \\
\hline 70 & 35.88 & 9928 & 97.51 & 74.46 \\
\hline 80 & 41.60 & 10036 & 98.57 & 78.19 \\
\hline 90 & 46.80 & 10098 & 99.18 & 1885703 \\
\hline
\end{tabular}


Table 10. contd...

\begin{tabular}{|c|c|c|c|c|c|}
\hline \# of Modules & \% of Modules & \# of Error Changes & \% of Error Changes & LOC & 1996082 \\
\hline \hline 100 & 52.00 & 10136 & 99.55 & 2029362 & 87.37 \\
\hline 110 & 57.20 & 10162 & 99.80 & 2085500 & 88.82 \\
\hline 120 & 62.40 & 10173 & 99.91 & 2106066 & 91.28 \\
\hline 129 & 67.08 & 10182 & 100.00 & 92.18 \\
\hline
\end{tabular}

Table 11. Distribution of Accumulative Percentage of Non-Error Changes and LOC for the Modules

\begin{tabular}{|c|c|c|c|c|c|}
\hline \# of Modules & $\%$ of Modules & \#of Non-Error Changes & $\%$ of Non-Error Changes & LOC & $\%$ of LOC \\
\hline 10 & 5.20 & 6145 & 66.53 & 597260 & 26.14 \\
\hline 20 & 10.40 & 7174 & 77.67 & 688207 & 30.12 \\
\hline 30 & 15.60 & 7820 & 84.66 & 1020145 & 44.65 \\
\hline 40 & 20.80 & 8286 & 89.70 & 1113032 & 48.72 \\
\hline 50 & 26.00 & 8605 & 93.16 & 1352786 & 59.21 \\
\hline 60 & 31.20 & 8811 & 95.39 & 1434835 & 62.80 \\
\hline 70 & 36.40 & 8963 & 97.03 & 1460835 & 63.94 \\
\hline 80 & 41.60 & 9068 & 98.17 & 1520795 & 66.56 \\
\hline 90 & 46.80 & 9144 & 98.99 & 1895540 & 82.97 \\
\hline 100 & 52.00 & 9198 & 99.58 & 1942151 & 85.01 \\
\hline 110 & 57.20 & 9225 & 99.87 & 1968230 & 86.15 \\
\hline 120 & 62.40 & 9235 & 99.98 & 2025147 & 88.64 \\
\hline 122 & 63.44 & 9237 & 100.00 & 2026774 & 88.71 \\
\hline
\end{tabular}

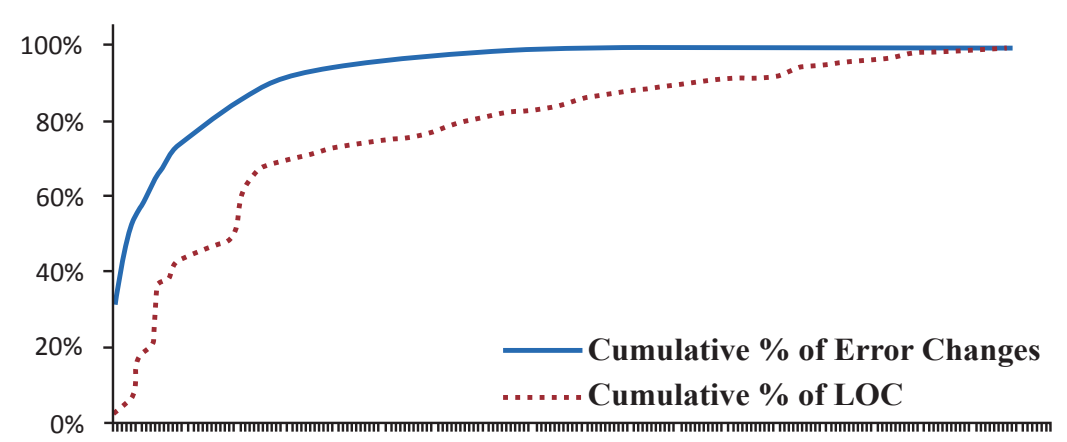

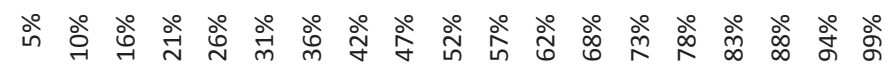

\section{$\%$ of Modules}

Fig. (6). Cumulative percentage of error changes and LOC versus the accumulative percentage of modules.

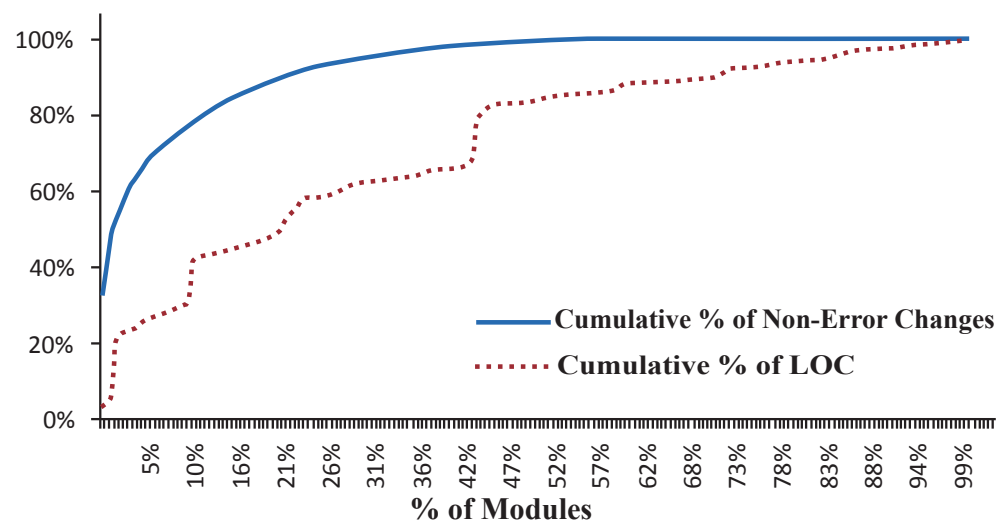

Fig. (7). Cumulative percentage of non-error changes and LOC versus the accumulative percentage of modules. 
Table 12. Distribution of Accumulative Percentage of Changes and LOC in Files

\begin{tabular}{|c|c|c|c|c|c|}
\hline \# of Files & \% of Files & \# of Changes & \% of Changes & LOC & 649563 \\
\hline \hline 1696 & 5.00 & 30149 & 60.48 & 937014 & 17.18 \\
\hline 3391 & 10.00 & 38443 & 77.11 & 1000427 & 24.79 \\
\hline 3871 & 11.42 & 39883 & 80.00 & 1216662 & 26.46 \\
\hline 5086 & 15.00 & 42911 & 86.08 & 1411207 & 32.19 \\
\hline 6781 & 20.00 & 45820 & 91.91 & 1538897 & 47.33 \\
\hline 8476 & 25.00 & 47515 & 95.31 & 1736287 & 40.71 \\
\hline 10172 & 30.00 & 49211 & 98.71 & 100.00 & 45.93 \\
\hline 10814 & 31.90 & 49853 & 47.53 \\
\hline
\end{tabular}

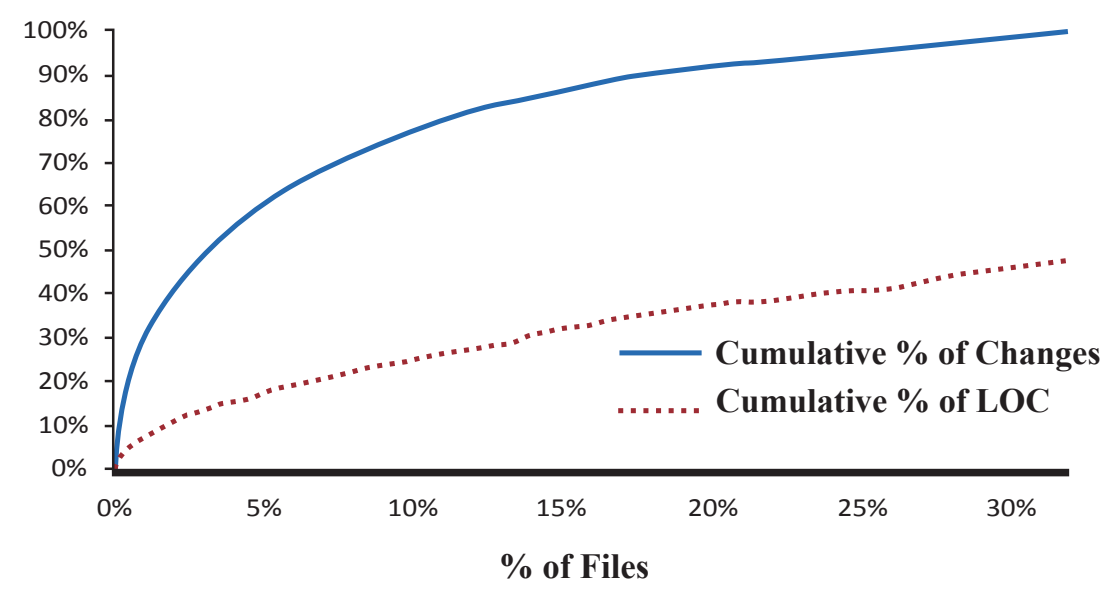

Fig. (8). Accumulative percentage of changes and LOC versus the accumulative percentage of files.

We sorted the modules in the descending order with respect to the number of changes. Fig. (5) shows the plot with the accumulative percentage of changes and LOC on the $y-$ axis with respect to the accumulative percentage of modules on the x-axis. Visually, we see that the two curves were fairly close and their trends were similar. Therefore, we accepted Hypothesis \#5 for modules.

When changes were examined in two groups: non-error changes and error changes, the phenomenon that we observed for total changes disappeared. Table $\mathbf{1 0}$ and $\mathbf{1 1}$ summarize the module LOC data for the error changes and nonerror changes. Fig. (6 and 7) are the plots of the data in Table 10 and 11. In both Fig. (6 and 7), the distance between the two curves was big and the trends between them were not similar. For error changes and non-error changes, we observed different behavior from that of total changes in terms of the accumulative percentage of modules and accumulative percentage of LOC with respect to the accumulative percentage of the changes.

\subsection{LOC for Files}

Table 12 summarizes the LOC data for files. We sorted the files and LOC for the files in the descending order with respect to the number of changes. Fig. (6) plotted the accumulative percentage of changes and LOC on the y-axis with respect to the accumulative percentage of files on the $\mathrm{x}$-axis. We plotted the graph for 10,814 files (about $32 \%$ of the total), because these files contained $100 \%$ of the changes.
We see in Fig. (8) that the two curves were not close and the trends were not similar. The gap between the blue and red lines was too big to accept Hypothesis $\# 5$ at the file level.

For Hypothesis \#5, we had the mixed results: At the module level, we accepted it only for total changes, not for error and non-error changes. At the file level, we rejected it at the total change level. Once again, we noticed the different behavior between modules and files for the total changes. More discussion on the mixed results is presented in the Discussion of the Findings section.

The conclusion on modules for the total changes seems to contradict the conclusion from that of Hypothesis \#4 test. A more detailed discussion on this issue is in the Discussion of the Findings section.

\section{DISCUSSION AND CONCLUSION}

Finding the power-law distribution, also known as the Pareto principle, as a common phenomenon in the change history of the large object-oriented system in its post-release evolution phase was not surprising. What was unexpected is that the power-law distribution was sporadic at the file level, in contrast to the finding at the module level. This finding by and large agrees with previous research results from [8-10].

Another unexpected finding was the difference between module and file in the relationship between size and the total 
changes. We found that the few modules that had the majority of changes $(>80 \%)$ contained the majority of code size $(>70 \%)$. However, the same cannot be said for the files.

These expected and unexpected findings suggest that in large object-oriented systems that are in their post-release evolution phase, the change behavior of the software artifacts is different for modules and files, and is likely to be more predictable at the module level than at the file level.

We found no correlation between the artifact size and changes. This finding goes against the majority opinion that the two are correlated $[5,9,10,12,19,20]$. On the surface, it seems to contradict our own finding that modules, which had the most code size contained the majority of changes. For correlation to exist between two groups of data they must be in synchronization with their increasing and decreasing trends, that is, when one group goes up (or down) in value, the other group must follow suit. This trend apparently did not exist in the data that we analyzed at either the module or file level. However, if we ignore the synchronization aspect of the trend, we did find that, at the module level, the total changes concentrated in a few modules that had the most code size. This finding suggests that the evolutionary changes in large object-oriented systems may not be sensitive to correlation.

Emam and colleagues found the confounding factor of class size in using metrics to predict various factors in software systems [12]. Since most Java files that we studied contained only one class, we considered the file and class to be the same artifact. Our findings showed mixed results. On one hand, at the module level, the total changes concentrated in a few modules that made up the bulk of the system size, although correlation between the two did not exist. On the other hand, the same cannot be said for error changes or nonerror changes at the module level; and no such phenomenon was observed for files for any kind of changes. The practical implication of our research findings is that the artifact size alone will unlikely to be an effective predictor for changeprone artifacts for files/classes.

We want to emphasize that all the suggestions and conclusions from our research apply to large object-oriented systems that are in their post-release evolution phase. Large systems that are in their development phase may exhibit different patterns in changes and in the relationship between artifact size and changes.

We have analyzed the maintenance change history of the Eclipse Project that consists of 220 modules (Java packages), 33904 files, 3780201 lines of code, and 49853 changes. We investigated two levels of software artifacts: module and file. At the module level, we found that the power-law distribution was a common phenomenon in total changes, error changes, non-error changes, and for all but one change categories. At the file level, the power-law distribution existed in total changes, error changes, non-error changes; however, the majority of the change categories (about two third) did not show power-law distribution.

For the relationship between artifact size and changes, we found that at the module level, a few modules that had the majority of the total changes accounted for the majority of the code size; however, this phenomenon did not exist for error changes and non-error changes. At the file level, the few files that accounted for the majority of total changes did not account for the majority of code size. We did not find any correlation between artifact size and any kind of changes at either the module or file level. Our findings cast doubt that artifact size alone can predict the change probability for individual files and modules.

Our research findings suggest that in large objectoriented systems, change-proneness prediction will likely be effective at module level for large object-oriented systems that are in their post-release evolution phase.

\section{VALIDITY THREATS}

There are some limitations in our research. We summarize these limitations as the internal and external threats.

Internal Threats: We collected the change data from the Eclipse Project's public repositories: CVS and Bugzilla. Any changes that were not logged in the two sources were not considered in the study. We make no claim about the accuracy of the data logged in these two sources. The metrics data were collected by using RSM-a commercial metrics tool, - We do not make any claims about the accuracy of the tool, but we believe that the tool collected the data consistently.

The manual classification process is subjective. We classified the changes into 19 categories by using our judgment. In the case where a change might be placed in multiple categories, we used our best judgment to place the change in the most suitable category.

External Threats: The Eclipse Project is a mature industrial software tool that has been in the post-release evolution process for more than a decade. The conclusions from this research are applicable to similar systems: large objectoriented systems that have been released and are in their post-release evolution phase. We do not suggest generalizing our research results to systems that are under development, because we believe that systems under development exhibit different change patterns and different relationship between changes and module/file.

\section{CONFLICT OF INTEREST}

The authors confirm that this article content has no conflicts of interest.

\section{ACKNOWLEDGEMENT}

Declared none.

\section{REFERENCES}

[1] M. Fowler, K. Beck, J. Brant, W. Opdyke, and D. Roberts, Refactoring: improving the design of existing code, Addison Wesley: USA, 1999.

[2] V.R. Basili, and B.T. Perricone, "Softw Errors and Complexity: An Empirical Investigation,” Commun. ACM, vol. 27, No. 1, pp. 42-52, 1984.

[3] V. Y. Shen, T. Yu, S. M. Thebaut, and L. R. Paulsen, "Identlfylng error-prone software: an empirical study," IEEE Trans. Softw., Eng., pp. 317-324, 1985

[4] C. Withrow, "Bug density and size in ada software," IEEE Softw., vol. 7,1, pp. 26-30, Jan/Feb. 1990. 
[5] K.H. Moller, and D.J. Paulish, "An empirical investigation of software fault distribution," Proc., First Int'l Softw. Metrics Symp. (Metrics '93), pp. 82-90, 1993

[6] N. Ohlsson, H. Alberg, "Predicting fault-prone software modules in telephone switches," IEEE Trans. Softw. Eng, vol. 22, no. 12, pp. 886-894, 1996.

[7] L. Hatton, "Reexamining the fault density component size connection," IEEE Softw., pp. 89-97, 1997.

[8] N. E. Fenton and N. Ohlsson, "Quantitative analysis of faults and failures in a complex software system," IEEE TSE, vol. 26, no. 8, 2000.

[9] T. J. Ostrand, E.J. Weyuker, and R. M. Bell, "Where the bugs are," In: Proc. ACM/International Symposium of Software Testing and Analysis, Boston: USA, pp. 86-96, July 2004.

[10] C. Andersson and P. Runeson, "A replicated quantitative analysis of fault distribution of complex software systems," IEEE Trans. Softw. Eng., vol. 33, no. 5, pp. 273-286, 2007.

[11] H. Zhang, "On the distribution of faults," IEEE Trans. Softw. Eng., vol 32, no. 2, pp. 301-302, 2008.

[12] K. El Emam, S. Benlarbi, and S.N. Rai, "The confounding effect of class size on the validity of object-oriented metrics," IEEE Trans. Softw. Eng., vol. 27, no.7, pp.630-650.

[13] IEEE standard, "IEEE standard classification for software anomalies," IEEE Std 1044-2009 (Revision of IEEE Std 1044-1993), pp. C1-15, 2010.

[14] Z. Li, L. Tan, X. Wang, S. Lu, Y. Zhou and C. Zhai, "Have things changed now? - an empirical study of bug characteristics in modern open source software," In: ACM/Architectural Support for Programming Languages and Operating Systems, San Jose: California, pp. 25-33, 2006.

[15] D. Lo, H. Cheng, J. Han, S. C. Khoo, and C. Sun, "Classification of software behaviors for failure detection: a discriminative pattern mining approach," In: Proc. ACM/International conference on knowledge discovery and data mining, Paris: France, 2009.

[16] K. Pan, S. Kim, and E.J. Whitehead, "Bug Classification Using Program Slicing Metrics," In: IEEE/International Workshop on Source Code Analysis and Manipulation, Philadelphia: USA, pp. 31-42, 2006.

[17] G. Soares, R. Gheyi, T. Massoni, M. Corn'elio, and D. Cavalcanti, "Generating unit tests for checking refactoring safety," $S B L P$, pp. 159-172, 2009.
[18] C. Görg, and P. Weißgerber, "Error detection by refactoring reconstruction," the 2005 International Workshop on Mining Software Repositories, MSR 2005, Saint Louis, Missouri, ACM: USA, 2005.

[19] Z. Qin, "Naive Bayes Classification Given Probability Estimation Trees, In: "Fifth International Conference on Machine Learning and Applications (ICMLA), Orlando: FL, pp. 34-42, 2006.

[20] J. Thorstern, "A Probabilistic Analysis of the Rocchio Algorithm with TFIDF for Text Categorization," In: Fourteenth International Conference on Machine Learning (ICML), Nashvlle, T.ennessee: USA, pp.143-151, 1997.

[21] CVS and Eclipse, Available at: http://www.grapeinfo.com/doc/win2000srv/filemanagement/cvs.html

[22] Boris Beizer, "Software Testing Techniques", $2^{\text {nd }}$ ed. The Coriolis Group: USA, 1990.

[23] P. Wolfgang: "Text Classification Tools Version 0.11". Available on:

http://www.cis.temple.edu/ wolfgang/Text\%20Classification $\% 20 \mathrm{~T}$ ools.pdf [last accessed $15^{\text {th }}$ October, 2012].

[24] A. G. Bluman, "Elementary Statistics: A Step by Step Approach", $2^{\text {nd }}$ ed. Wm. C. Brown Publishers: Dubuque, Iowa: USA, 1995.

[25] Potanin, J. Noble, M. R. Frean, and R. Biddle., "Scale-free geometry in object-oriented programs," $A C M$ Commun., vol. 48, no. 5, 2005.

[26] R. Wheeldon and S. Counsell, "Power law distribution in class relationships," In: IEEE/International Workshop on Source Code Analysis and Manipulation, Computer Soc. Press: Los Alamitos, pp. 45-54, 2003.

[27] L. Hatton, "Power-Law Distributions of Component Size in General Software Systems," In: IEEE Trans Softw. Eng., vol. 35, no. 4 pp. 566-572, 2009.

[28] J.M. Juran, F.M. Gryna, Jr., and F.M. Bingham, "Quality Control Handbook", $3^{\text {rd }}$ ed, McGraw Hill: New York, 1979.

[29] I.Timea, and D. Barbara, "The vital few and trivial many: An empirical analysis of the pareto distribution of defects," Proc. Lecture Notes in Informatics, pp.151-162, Nov, 2010.

[30] Matlab Official Website, Available at: http://www.mathworks.com/products/matlab/

[31] Aaron, S. R. Cosma, and M.E.J. Newman, "Power-Law Distribution in Empirical Data,” Soc. Ind. Appl. Math., vol. 51, no. 4, 2009.

Received: February 02, 2013

Revised: February 12, 2012

Accepted: February 16, 2012

(C) Tiwari et al.; Licensee Bentham Open.

This is an open access article licensed under the terms of the Creative Commons Attribution Non-Commercial License (http://creativecommons.org/licenses/by-nc/3.0/) which permits unrestricted, non-commercial use, distribution and reproduction in any medium, provided the work is properly cited. 